N. Obata

Nagoya Math. J.

Vol. 118 (1990), 111-132

\title{
A CHARACTERIZATION OF THE LÉVY LAPLACIAN IN TERMS OF INFINITE DIMENSIONAL ROTATION GROUPS
}

\author{
NOBUAKI OBATA
}

\section{Introduction}

P. Lévy introduced, in his celebrated books [21] and [22], an infinite dimensional Laplacian called the Lévy Laplacian in connection with a number of interesting topics in variational calculus. One of the most significant features of the Lévy Laplacian is observed when it acts on the singular part of the second functional derivatives. For this reason the Lévy Laplacian has become important also in white noise analysis initiated by $\mathrm{T}$. Hida [12]. On the other hand, as was pointed out by $\mathrm{H}$. Yoshizawa [29], infinite dimensional rotation groups are profoundly concerned with the structure of white noise, and therefore, play essential roles in certain problems of stochastic calculus. Motivated by these works, we aim at developing harmonic analysis on infinite dimensional spaces by means of the Lévy Laplacian and infinite dimensional rotation groups.

The significant property of the Lévy Laplacian mentioned above appears typically when it acts on particular quadratic functions on infinite dimensional spaces. This fact leads us naturally to the notion of normal functions which will be taken as the domain of the Lévy Laplacian, and thereby we find many characteristic properties of the Lévy Laplacian. The main purpose of this paper is to investigate the structure of the rotation group describing the invariance of the Lévy Laplacian and to give its characterization by means of this invariance. Our main results are stated in Sections 3 and 4.

Here is a summary of the paper.

Throughout the paper $H$ denotes the real Hilbert space $L^{2}(T, \nu)$ with $(T, \nu)$ being a probability space satisfying some conditions prescribed in Section 1 . The Lévy Laplacian $\Delta$ is defined by

Received December 26, 1988. 


$$
\Delta F(\xi)=\lim _{N \rightarrow \infty} \frac{1}{N} \sum_{n=1}^{N}\left\langle F^{\prime \prime}(\xi) e_{n}, e_{n}\right\rangle
$$

where $\left\{e_{n}\right\}_{n=1}^{\infty}$ is an equally dense CONS (= complete orthonormal sequence) in $H$ and $F$ is a normal function on $H$ introduced below. It will be noted that the limit always exists and does not depend upon the choice of such a CONS.

We now introduce normal functions which will play an essential role in the study of the Lévy Laplacian. Let $\mathscr{M}$ be the set of all multiplication operators $M(\varphi)$ with $\varphi \in L^{\infty}(T, \nu), \mathscr{K}$ the set of all compact operators on $H$ and put $\mathscr{A}=\mathscr{M}+\mathscr{K}$. It is known that $\mathscr{A}$ becomes a norm closed subalgebra of the algebra $\mathscr{B}=\mathscr{B}(H)$ of bounded operators on $H$. A normal polynomial on $H$ is by definition a member of the algebra generated by constant functions, linear functions and quadratic functions of the form $\langle A \xi, \xi\rangle, A \in \mathscr{A}$. Taking the completion of the normal polynomials with respect to the topology of uniform convergence on bounded subsets together with all the derivatives, we obtain the space $\mathfrak{R}$ of normal functions (see Section 1). It is noted that $\mathfrak{R}$ becomes a Fréchet space and is closed under pointwise multiplication.

As we mentioned before, the main purpose of this paper is to establish a relation between the Lévy Laplacian and infinite dimensional rotation groups. To our goal we first investigate the structure of the rotation group describing the invariance of the Lévy Laplacian. Set

$$
O(H ; \Delta)=\{g \in O(H) ; U(g) \Delta=\Delta U(g)\},
$$

where the action of the orthogonal group $O(H)$ on functions $F$ on $H$ is defined by

$$
(U(g) F)(\xi)=F\left(g^{-1} \xi\right), \quad \xi \in H, \quad g \in O(H) .
$$

It is very interesting that the structure of $O(H ; \Delta)$ is completely determined.

We now prepare some notation. The most important subgroup of $O(H)$ comes from transformations of the probability space $(T, \nu)$. With each transformation $\alpha$ of $T$ we associate a rotation $g_{\alpha} \in O(H)$ defined by the formula:

$$
\left(g_{\alpha} \xi\right)(t)=\left(\frac{d \nu\left(\alpha^{-1}(t)\right)}{d \nu(t)}\right)^{1 / 2} \xi\left(\alpha^{-1}(t)\right), \quad \xi \in H, t \in T
$$

The group $\mathfrak{I}(T)$ of all transformations is then regarded as a subgroup of 
$O(H)$. The subgroup of all measure-preserving transformations will be donoted by $\mathfrak{I}(T, \nu)$. Let $O_{c}(H)$ denote the subgroup of all rotations which are the identity modulo compact operators and we set $O_{a}(H)=O(H) \cap$ $\mathscr{M}$, which becomes a maximal abelian subgroup of $O(H)$. With these notations, we shall prove the following result in Section 3.

Theorem A (Invariance of the Lévy Laplacian). The rotation group $O(H ; \Delta)$ admits a factorization into a semidirect product of three subgroups:

$$
O(H ; d)=\mathfrak{I}(T, \nu) \ltimes\left(O_{a}(H) \ltimes O_{c}(H)\right)=\left(\mathfrak{I}(T, \nu) \ltimes O_{a}(H)\right) \ltimes O_{c}(H) .
$$

The result suggests that $\mathfrak{I}(T, \nu)$ is indispensable to the analysis of the Lévy Laplacian and, in fact, we shall illustrate this in the problem of characterizing the Lévy Laplacian. While, it is also interesting that $O(H ; \Delta)$-invariant eigenfunctions of the Lévy Laplacian are determined using the subgroup $O_{c}(H)$ (Proposition 3.6).

We then discuss characterization of the Lévy Laplacian by means of the invariance described in Theorem A. To this end we notice the following properties of the Lévy Laplacian.

(L1) $\Delta$ is a continous linear operator from $\mathfrak{N}$ into itself;

(L2) $\Delta$ is a derivation on $\mathfrak{R}$, i.e., for any $F_{1}, F_{2} \in \mathfrak{R}$,

$$
\Delta\left(F_{1} F_{2}\right)=\Delta F_{1} \cdot F_{2}+F_{1} \cdot \Delta F_{2} ;
$$

(L3) $\triangle$ annihilates linear functions;

(L4) for any non-negative quadratic function $F(\xi)=\langle A \xi$, $\xi\rangle$ with $A \in \mathscr{A}, \Delta F(\xi)$ is a non-negative constant.

The proofs of (L1) and (L2) are given in Section 1. On the other hand, (L3) and (L4) are immediate consequences from the definition. Finally, by Theorem $\mathrm{A}$ we have

(L5) $\Delta$ is invariant under $\mathfrak{T}(T, \nu)$.

Among others, properties (L2) and (L5) are very noticeable, and thereby a clear discrimination is made between the Lévy Laplacian and another Laplacians on infinite or finite dimensional spaces. We prove in Section 4 the following assertion.

Theorem B (Characterization of the Lévy Laplacian). If an operator on $\mathfrak{R}$ satisfies the properties (L1)-(L5), it is a constant multiple of the Lévy Laplacian.

In Section 5 we establish a formula which links the Lévy Laplacian 
and asymptotic spherical mean. A noteworthy feature of normal functions is observed here again (Proposition 5.1).

In Appendix we construct CONS's which are equally dense independent of the arrangement and discuss rearrangement of equally dense CONS's in general. The last topic is somewhat related to a similar problem in uniformly distributed sequences ([25]).

AckNowledgements. I acknowledge with special thanks stimulations that came from Professors K. Aomoto, T. Hirai, H. Nomoto and $H$. Yoshizawa. Most cordial thanks are due to Professor T. Hida whose influence on my development and direction has been both beneficial and far-reaching.

\section{§1. The Lévy Laplacian and normal functions}

Let $T$ be a separable complete metric space and $\nu$ a Borel probability measure on it. Let $H$ be the real Hilbert space $L^{2}(T, \nu)$ with the inner product $\langle\cdot, \cdot\rangle$ and the norm $\|\cdot\|$. In order to avoid inessential argument we assume that the measure space $(T, \nu)=(T, \mathscr{T}, \nu)$ is complete. We further put the following two assumptions on $(T, \nu)$ :

(A1) every non-empty open subset of $T$ has positive measure;

(A2) the probability space $(T, \nu)$ admits no atoms.

Let $\mathscr{B}=\mathscr{B}(H)$ denote the algebra of all bounded operators on $H$ equipped with the operator norm topology. With each $\varphi \in L^{\infty}(T, \nu)$ we associate a multiplication operator $M(\varphi) \in \mathscr{B}$ by

$$
(M(\varphi) \xi)(t)=\varphi(t) \xi(t), \quad \xi \in H, \quad t \in T .
$$

Let $\mathscr{M}$ be the set of such multiplication operators $M(\varphi)$ and $\mathscr{K}$ the set of compact operators on $H$. Note that (A2) is equivalent to

$\left(\mathrm{A} 2^{\prime}\right) \quad \mathscr{M} \cap \mathscr{K}=\{0\}$.

We set

$$
\mathscr{A}=\mathscr{M}+\mathscr{K}=\left\{M(\varphi)+K ; \varphi \in L^{\infty}(T, \nu), K \in \mathscr{K}\right\},
$$

which becomes a closed subalgebra of $\mathscr{B}$ (cf. [4, §1.8]).

As is well known, one of the most significant properties of the Lévy Laplacian is observed when it acts on particular quadratic functions on infinite dimensional spaces ([22]). These quadratic functions lead us the notion of normal functions which will be taken as the domain of the Lévy Laplacian. 
Definition. A function on $H$ is called a normal polynomial if it belongs to the algebra generated by constant functions, linear functions and quadratic functions of the form $\langle A \xi, \xi\rangle, A \in \mathscr{A}$. Let $\mathfrak{N}$ be the completion of the normal polynomials with respect to the topology of uniform convergence on bounded subsets in $H$ together with all the Fréchet derivatives. A member of $\mathfrak{N}$ is called a normal function.

Note that $\mathfrak{R}$ become a Fréchet space and remind that $\mathfrak{N}$ is closed under pointwise multiplication. In fact, we may prove a more general result below with no difficulty.

Proposition 1.1. Let $f$ be a $C^{\infty}$-function on $\boldsymbol{R}^{m}$. Then for any $F_{1}$, $\cdots, F_{m} \in \mathfrak{N}$ the function $F(\xi)=f\left(F_{1}(\xi), \cdots, F_{m}(\xi)\right)$ is again a normal function.

As usual we identify the derivatives $F^{\prime}(\xi)$ and $F^{\prime \prime}(\xi)$ with an element of $H$ and a symmetric operator in $\mathscr{B}$, respectively, by the formula:

$$
F(\xi+\eta)=F(\xi)+\left\langle F^{\prime}(\xi), \eta\right\rangle+\frac{1}{2}\left\langle F^{\prime \prime}(\xi) \eta, \eta\right\rangle+o\left(\|\eta\|^{2}\right)
$$

for $\xi \in H$ and small $\eta \in H$.

Proposition 1.2. If $F$ is a normal function, then $F^{\prime \prime}(\xi) \in \mathscr{A}$ for any $\xi \in H$. More precisely, for each $\xi \in H$ there exist unique $\varphi(\xi)=\varphi(\xi ; \cdot) \epsilon$ $L^{\infty}(T, \nu)$ and $K(\xi) \in \mathscr{K}$ such that

$$
F^{\prime \prime}(\xi)=M(\varphi(\xi))+K(\xi) .
$$

Proof. It is easy to see by a direct calculation that $F^{\prime \prime}(\xi) \in \mathscr{A}$ for any normal polynomial $F$. Since $\mathscr{A}$ is a closed subalgebra of $\mathscr{B}$, the same is true for arbitrary normal functions. The uniqueness of the expression (1-1) follows from $\left(\mathrm{A} 2^{\prime}\right)$.

Q.E.D.

Definition ([22, Part 3, §30]). A complete orthonormal sequence (=CONS) $\left\{e_{n}\right\}_{n=1}^{\infty}$ in $H=L^{2}(T, \nu)$ is called equally dense if

$$
\lim _{N \rightarrow \infty} \frac{1}{N} \sum_{n=1}^{N} \int_{T} \varphi(t) e_{n}(t)^{2} d \nu(t)=\int_{T^{\prime}} \varphi(t) d \nu(t),
$$

or equivalently,

$$
\lim _{N \rightarrow \infty} \frac{1}{N} \sum_{n=1}^{N}\left\langle M(\varphi) e_{n}, e_{n}\right\rangle=\int_{T} \varphi(t) d \nu(t)
$$


for any $\varphi \in L^{\infty}(T, \nu)$.

Generally speaking, the property of being equally dense depends upon the arrangement of the CONS. In this connection we mention a few results in Appendix. From now on we fix an equally dense CONS in $H$ and denote it by $\left\{e_{n}\right\}_{n=1}^{\infty}$.

Lemma 1.3. For any $F \in \mathfrak{N}$ we have

$$
\lim _{N \rightarrow \infty} \frac{1}{N} \sum_{n=1}^{N}\left\langle F^{\prime \prime}(\xi) e_{n}, e_{n}\right\rangle=\int_{T} \varphi(\xi ; t) d \nu(t),
$$

where $\varphi(\xi ; \cdot)$ is given by $(1-1)$.

Proof. By Proposition 1.2 we have $F^{\prime \prime}(\xi)=M(\varphi(\xi))+K(\xi)$, where $\varphi(\xi) \in L^{\infty}(T, \nu)$ and $K(\xi) \in \mathscr{K}$. Since $\left\{e_{n}\right\}_{n=1}^{\infty}$ is equally dense, we have

$$
\lim _{N \rightarrow \infty} \frac{1}{N} \sum_{n=1}^{N}\left\langle M(\varphi(\xi)) e_{n}, e_{n}\right\rangle=\int_{T} \varphi(\xi ; t) d \nu(t) .
$$

On the other hand, since $K(\xi)$ is compact,

$$
\lim _{n \rightarrow \infty}\left\langle K(\xi) e_{n}, e_{n}\right\rangle=0
$$

and therefore,

$$
\lim _{N \rightarrow \infty} \frac{1}{N} \sum_{n=1}^{N}\left\langle K(\xi) e_{n}, e_{n}\right\rangle=0 .
$$

Then (1-3) follows from (1-4) and (1-5).

Q.E.D.

We now come to the following

Definition. For a normal function $F \in \mathfrak{N}$ we put

$$
\Delta F(\xi)=\lim _{N \rightarrow \infty} \frac{1}{N} \sum_{n=1}^{N}\left\langle F^{\prime \prime}(\xi) e_{n}, e_{n}\right\rangle, \quad \xi \in H .
$$

(By Lemma 1.3 the limit always exists and is independent of the choice of an equally dense CONS.) The operator $\Delta$ is called the Lévy Laplacian.

Proposition 1.4. The Lévy Laplacian $\Delta$ is a continuous linear operator from $\mathfrak{N}$ into itself.

Proof. For brevity we put

$$
L(A)=\lim _{N \rightarrow \infty} \frac{1}{N} \sum_{n=1}^{N}\left\langle A e_{n}, e_{n}\right\rangle, \quad A \in \mathscr{A} .
$$


The limit always exists (cf. the proof of Lemma 1.3). Suppose that $F$ is a normal polynomial given by

$(1-7) \quad F(\xi)=P\left(\left\langle a_{1}, \xi\right\rangle, \cdots,\left\langle a_{l}, \xi\right\rangle,\left\langle A_{1} \xi, \xi\right\rangle, \cdots,\left\langle A_{m} \xi, \xi\right\rangle\right), \quad \xi \in H$,

where $a_{1}, \cdots, a_{l} \in H, A_{1}, \cdots, A_{m} \in \mathscr{A}$ and $P$ is a polynomial in $l+m$ variables. Then by a direct calculation, we obtain

$$
\Delta F(\xi)=\sum_{j=1}^{m} 2 L\left(A_{j}\right) \partial_{l+j} P .
$$

Hence $\Delta F$ is also a normal polynomial.

We next show the continuity. For any bounded set $B \subset H$ and nonnegative integer $n$ we put

$$
\|F\|_{B, n}=\sup \left\{\left\|F^{(n)}(\xi)\right\| ; \xi \in B\right\},
$$

where $\left\|F^{(n)}(\xi)\right\|$ is the usual norm of $n$-linear forms, i.e.,

$$
\left\|F^{(n)}(\xi)\right\|=\sup \left\{\left|F^{(n)}(\xi)\left(\xi_{1}, \cdots, \xi_{n}\right)\right| ;\left\|\xi_{1}\right\| \leqslant 1, \cdots,\left\|\xi_{n}\right\| \leqslant 1\right\} .
$$

The topology of $\mathfrak{R}$ is by definition given by the seminorms $\|\cdot\|_{B, n}$. A direct calculation implies that $\|\Delta F\|_{B, n} \leqslant\|F\|_{B, n+2}$ for any normal polynomial $F$. Since the normal polynomials form a dense subspace of $\mathfrak{R}$, the same inequality holds for any $F$ in $\Re$. Consequently, the Lévy Laplacian is a continuous linear operator from $\mathfrak{N}$ into itself.

Q.E.D.

Proposition 1.5. The Lévy Laplacian is a derivation, i.e., for any pair of normal functions $F_{1}$ and $F_{2}$ we have

$$
\Delta\left(F_{1} F_{2}\right)(\xi)=\Delta F_{1}(\xi) \cdot F_{2}(\xi)+F_{1}(\xi) \cdot \Delta F_{2}(\xi), \quad \xi \in H .
$$

This illustrates a striking contrast to the finite dimensional Laplacian. The proof is easy and omitted (see also Lemma 3.5).

Finally we remind that the normal functions cover many important functions on Hilbert space. For example, Hilbert-Schmidt polynomials ([1]), regularly analytic functions ([26]) and ordinary Brownian functionals through the $S$-transform ([18]) are normal functions. Moreover, the exponential function $F(\xi)=\exp \left(-\|\xi\|^{2}\right)$ which corresponds to a physically important Brownian functional is also a normal function.

\section{§ 2. Infinite dimensional rotation groups}

The group of all orthogonal operators (or rotations) on $H$ is denoted 
by $O(H)$ and equipped with the norm topology. Subgroups of $O(H)$ are generally called (infinite dimensional) rotation groups.

The most important subgroup of $O(H)$ arises from transformations of $T$. A transformation on $(T, \nu)$ is by definition a measurable automorphism $\alpha$ on $T$ such that $\nu \circ \alpha^{-1}$ and $\nu$ have the same class of null sets. A transformation $\alpha$ is called measure-preserving if $\nu \circ \alpha^{-1}=\nu$. Let $\mathfrak{T}(T)$ and $\mathfrak{I}(T, \nu)$ denote the group of all transformations and its subgroup of measure-preserving ones, respectively. With each $\alpha \in \mathfrak{I}(T)$ we associate a rotation $g_{\alpha} \in O(H)$ by the formula:

$$
\left(g_{\alpha} \xi\right)(t)=\left(\frac{d \nu\left(\alpha^{-1}(t)\right)}{d \nu(t)}\right)^{1 / 2} \xi\left(\alpha^{-1}(t)\right), \quad t \in T, \quad \xi \in H .
$$

By means of the mapping $\alpha \rightarrow g_{\alpha}$ we identify $\mathfrak{I}(T)$ with a subgroup of $O(H)$ which will be denoted by the same symbol.

We put

$$
O_{c}(H)=\{g \in O(H) ; I-g \in \mathscr{K}\},
$$

where $I$ denotes the identity operator on $H$, and

$$
O_{a}(H)=O(H) \cap \mathscr{M} .
$$

Note that $O_{c}(H)$ is a normal subgroup of $O(H)$. Note also an obvious relation

$$
g_{a} M(\varphi) g_{\alpha}^{-1}=M\left(\varphi \circ \alpha^{-1}\right), \quad g_{\alpha} \in \mathfrak{I}(T), \quad \varphi \in L^{\infty}(T, \nu) .
$$

Then we have the following assertion.

Lemma 2.1. The three rotation groups $\mathfrak{I}(T), O_{a}(H)$ and $O_{c}(H)$ constitute a semidirect product: $\mathfrak{I}(T) \ltimes\left(O_{a}(H) \ltimes O_{c}(H)\right)=\left(\mathfrak{I}(T) \ltimes O_{a}(H)\right)$ $\ltimes O_{c}(H)$.

We next show that the semidirect product is the automorphism group of $\mathscr{A}: O(H ; \mathscr{A})=\left\{g \in O(H) ; g \mathscr{A} g^{-1}=\mathscr{A}\right\}$, namely

Proposition 2.2. It holds that

$$
O(H ; \mathscr{A})=\mathfrak{I}(T) \ltimes\left(O_{a}(H) \ltimes O_{c}(H)\right)=\left(\mathfrak{I}(T) \ltimes O_{a}(H)\right) \ltimes O_{c}(H) .
$$

Before we come to the proof some preliminary results are given.

LEMMA 2.3. Let $\Psi$ be an algebra automorphism of the Banach algebra $L^{\infty}(T, \nu)$. Then there exists a unique $\alpha \in \mathfrak{I}(T)$ such that $\Psi(\varphi)=\varphi \circ \alpha^{-1}$ for any $\varphi \in L^{\infty}(T, \nu)$. 
Lemma 2.4. If $A \in \mathscr{B}$ commutes with every multiplication operator $M \in \mathscr{M}$ modulo compact operators, i.e., $A M-M A \in \mathscr{K}$ for any $M \in \mathscr{M}$, then $A$ belongs to $\mathscr{A}=\mathscr{M}+\mathscr{K}$.

These are easily verified with the help of the corresponding results for complex algebras, see [3], [17] and [28].

Lemma 2.5. For $g \in O(H)$ the following two conditions are equivalent:

(i) $g M g^{-1}-M \in \mathscr{K}$ for any $M \in \mathscr{M}$;

(ii) $g$ belongs to $O_{a}(H) \ltimes O_{c}(H)$.

Proof. The implication (ii) $\Rightarrow$ (i) is obvious. We show that (i) implies (ii). Now suppose that $g M g^{-1}-M \in \mathscr{K}$ for any $M \in \mathscr{M}$. Then by Lemma 2.4, $g$ belongs to $\mathscr{M}+\mathscr{K}$, say, $g=M+K$. Since

$$
I={ }^{t} g g=\left({ }^{t} M+{ }^{t} K\right)(M+K)={ }^{t} M M+{ }^{t} M K+{ }^{t} M K+{ }^{t} K K,
$$

$I-{ }^{t} M M={ }^{t} M K+{ }^{t} K M+{ }^{t} K K$ belongs $\mathscr{M} \cap \mathscr{K}=\{0\} . \quad$ Hence $M$ belongs $O_{a}(H)$ and ${ }^{t} M g={ }^{t} M(M+K)=I+{ }^{t} M K$ belongs to $O_{c}(H)$. Consequently, we obtain the factorization $g=M\left(I+{ }^{t} M K\right) \in O_{a}(H) \ltimes O_{c}(H)$. Q.E.D.

Proof of Proposition 2.2. It is easily seen that any rotation $g \in \mathfrak{I}(T)$ $\ltimes\left(O_{a}(H) \ltimes O_{c}(H)\right)$ induces an automorphism of $\mathscr{A}$. We shall prove the converse assertion. Suppose that $g \in O(H)$ induces an automorphism of $\mathscr{A}$. In particular, $g \mathscr{M} g^{-1}+\mathscr{K}=\mathscr{M}+\mathscr{K}$. Hence $g$ induces an automorphism $\hat{g}$ of $L^{\infty}(T, \nu)$ determined uniquely by the condition that

$$
g M(\varphi) g^{-1}-M(\hat{g}(\varphi)) \in \mathscr{K}, \quad \varphi \in L^{\infty}(T, \nu) .
$$

By Lemma 2.3, there exists a transformation $\alpha \in \mathfrak{I}(T)$ such that

$$
\hat{g}(\varphi)=\varphi \circ \alpha^{-1}, \quad \varphi \in L^{\infty}(T, \nu) .
$$

In view of (2-2), (2-3) and (2-4) we have

$$
g M(\varphi) g^{-1}-g_{\alpha} M(\varphi) g_{\alpha}^{-1} \in \mathscr{K} .
$$

Hence, by Lemma 2.5, we have $g_{\alpha}^{-1} g \in O_{a}(H) \ltimes O_{c}(H)$. Consequently, $g$ belongs to $\mathfrak{T}(T) \ltimes\left(O_{a}(H) \ltimes O_{c}(H)\right)$.

Q.E.D.

\section{§3. Invariance of the Lévy Laplacian}

In this section we find a very noticeable result which makes us possible to study the Lévy Laplacian from the viewpoint of harmonic analysis. Namely, we determine the structure of the rotation group 
describing the invariance of the Lévy Laplacian:

$$
O(H ; \Delta)=\{g \in O(H) ; U(g) \Delta=\Delta U(g)\},
$$

where an operator $U(g)$ on functions $F$ on $H$ by

$$
(U(g) F)(\xi)=F\left(g^{-1} \xi\right), \quad \xi \in H .
$$

The main result is the following

TheOREM A (Invariance of the Lévy Laplacian). The rotation group $O(H ; \Delta)$ admits a factorization into a semidirect product of three subgroups:

$$
O(H ; \Delta)=\mathfrak{I}(T, \nu) \ltimes\left(O_{a}(H) \ltimes O_{c}(H)\right)=\left(\mathfrak{I}(T, \nu) \ltimes O_{a}(H)\right) \ltimes O_{c}(H) .
$$

It is interesting and important that the invariance of the Lévy Laplacian is completely described with the three rotation groups each of which is structurally very well known. Furthermore, the factorization of $O(H ; \Delta)$ suggests particular importance of $\mathfrak{I}(T, \nu)$. In fact, we shall give a group-theoretical characterization of the Lévy Laplacian in the next section. For the proof we begin with the following

Lemma 3.1. For a rotation $g \in O(H)$ the following two conditions are equivalent:

(i) $U(g) \mathfrak{N} \subset \mathfrak{N}$; (ii) $g \mathscr{A} g^{-1} \subset \mathscr{A}$.

Proof. First we suppose (ii). Let $F$ be a normal polynomial given as in (1-7). Then

$$
\begin{aligned}
& (U(g) F)(\xi)=F\left(g^{-1} \xi\right)=F\left({ }^{t} g \xi\right)= \\
& \quad=P\left(\left\langle g a_{1}, \xi\right\rangle, \cdots,\left\langle g a_{l}, \xi\right\rangle,\left\langle g A_{1} g^{-1} \xi, \xi\right\rangle, \cdots,\left\langle g A_{m} g^{-1} \xi, \xi\right\rangle\right) .
\end{aligned}
$$

Hence, by the assumption (ii) we see that $U(g) F$ is also a normal polynomial. Since $U(g)$ is a continuous operator on $C_{b}^{\infty}(H)$, the assertion (i) follows. Here $C_{b}^{\infty}(H)$ is equipped with the seminorms $\|\cdot\|_{B, n}$ defined in $(1-8)$.

We next show that (i) implies (ii). Consider a normal function $F(\xi)$ $=\langle A \xi, \xi\rangle$ with $A \in \mathscr{A}$. Then, by assumption $U(g) F$ is also normal. Hence $(U(g) F)^{\prime \prime}(\xi)=2 g A g^{-1}$ belongs to $\mathscr{A}$ by Proposition 1.2. Consequently, $g \mathscr{A} g^{-1} \subset \mathscr{A}$.

Q.E.D.

LEMMA 3.2. For a rotation $g \in O(H), U(g) \mathfrak{N}=\mathfrak{N}$ holds if and only if $g \in O(H ; \mathscr{A})$.

Proof. Immediate from Lemma 3.1.

Q.E.D. 
LEMma 3.3. Let $\alpha \in \mathfrak{T}(T)$. Then $U\left(g_{\alpha}\right) \Delta=\Delta U\left(g_{\alpha}\right)$ holds if and only if $\alpha \in \mathfrak{I}(T, \nu)$.

Proof. Let $F \in \mathfrak{R}$ with $F^{\prime \prime}(\xi)=M(\varphi(\xi))+K(\xi)$. Then by Lemma 1.3 we obtain

$$
\left(U\left(g_{\alpha}\right) \Delta F\right)(\xi)=\int_{T} \varphi\left(g_{\alpha}^{-1} \xi ; t\right) d \nu(t) .
$$

On the other hand, noting (2-2) we have

$$
\left(U\left(g_{\alpha}\right) F\right)^{\prime \prime}(\xi)=g_{\alpha} F^{\prime \prime}\left(g_{\alpha}^{-1} \xi\right) g_{\alpha}^{-1}=M\left(\varphi\left(g_{\alpha}^{-1} \xi\right) \circ \alpha^{-1}\right)+g_{\alpha} K\left(g_{\alpha}^{-1} \xi\right) g_{\alpha}^{-1}
$$

and therefore,

$$
\begin{aligned}
\left(\Delta U\left(g_{\alpha}\right) F\right)(\xi) & =\int_{T} \varphi\left(g_{\alpha}^{-1} \xi ; \alpha^{-1}(t)\right) d \nu(t) \\
& =\int_{T} \varphi\left(g_{\alpha}^{-1} \xi ; t\right) \frac{d \nu(\alpha(t))}{d \nu(t)} d \nu(t) .
\end{aligned}
$$

If $\alpha \in \mathfrak{I}(T, \nu)$, apparently (3-1) and (3-2) are in coincidence. Conversely, suppose that (3-1) coincides with (3-2) for any $F \in \mathfrak{R}$. Then, taking $F(\xi)$ $=\frac{1}{2}\langle M(\varphi) \xi, \xi\rangle, \varphi \in L^{\infty}(T, \nu)$, we have

$$
\int_{T} \varphi(t) d \nu(t)=\int_{T} \varphi(t) \frac{d \nu(\alpha(t))}{d \nu(t)} d \nu(t), \quad \varphi \in L^{\infty}(T, \nu) .
$$

This means that $\alpha \in \mathfrak{I}(T, \nu)$.

Q.E.D.

Proof of Theorem A. It follows from Lemma 3.2 that $O(H ; \Delta) \subset$ $O(H ; \mathscr{A})$. Then, with the help of Lemma 3.3 we need only to show that $U(g) \Delta=\Delta U(g)$ for any $g \in O_{a}(H) \ltimes O_{c}(H)$. Let $F \in \Re$ with $F^{\prime \prime}(\xi)=M(\varphi(\xi))$ $+K(\xi), \varphi(\xi) \in L^{\infty}(T, \nu), K(\xi) \in \mathscr{K}$. Then, for any $g \in O_{a}(H) \ltimes O_{c}(H)$ we have

$(U(g) F)^{\prime \prime}(\xi)=g F^{\prime \prime}\left(g^{-1} \xi\right) g^{-1}=g\left(M\left(\varphi\left(g^{-1} \xi\right)\right)+K\left(g^{-1} \xi\right)\right) g^{-1}=M\left(\varphi\left(g^{-1} \xi\right)\right)+K^{\prime}$ with some $K^{\prime} \in \mathscr{K}$. It then follows from Lemma 1.3 that

$$
(\Delta U(g) F)(\xi)=\int_{T} \varphi\left(g^{-1} \xi ; t\right) d \nu(t)=\Delta F\left(g^{-1} \xi\right)=(U(g) \Delta F)(\xi) .
$$

This completes the proof.

Q.E.D.

Remark 3.4. There arises a natural question of translation invariance of the Lévy Laplacian. However, this is not so interesting as rotation 
invariance because the Lévy Laplacian is invariant under arbitrary translations on $H$ in the sense that $\Delta V(\eta)=V(\eta) \Delta$ for any $\eta \in H$, where an operator $V(\eta)$ is defined as

$$
(V(\eta) F)(\xi)=F(\xi-\eta), \quad \xi \in H .
$$

The proof is straightforward.

As a simple application of Theorem A, we investigate $O(H ; \Delta)$-invariant eigenfunctions of the Lévy Laplacian. To this end we need the following lemma which generalizes Proposition 1.5. For the proof we only need to recall the definition of the Lévy Laplacian and the fact that $\lim _{n \rightarrow \infty}\left\langle a, e_{n}\right\rangle$ $=0$ for $a \in H$.

Lemma 3.5. Let $f$ be a $C^{\infty}$-function on $\boldsymbol{R}^{m}$ and let $F_{1}, \cdots, F_{m}$ be normal functions. Put $F(\xi)=f\left(F_{1}(\xi), \cdots, F_{m}(\xi)\right)$. Then

$$
\Delta F(\xi)=\sum_{j=1}^{m} \partial_{j} f\left(F_{1}(\xi), \cdots, F_{m}(\xi)\right) \cdot \Delta F_{j}(\xi) .
$$

Proposition 3.6. Any $O(H ; \Delta)$-invariant eigenfunction of $\Delta$ with eigenvalue $\lambda \in \boldsymbol{R}$ is of the form:

$$
F(\xi)=C \exp \left(\frac{\lambda}{2}\|\xi\|^{2}\right),
$$

where $C \in \boldsymbol{R}$ is a constant.

Proof. Suppose that $F \in \mathfrak{R}$ is invariant under $O(H ; \Delta)$. Then $F$ is invariant under $O_{c}(H)$ by Theorem A and therefore, we may write

$$
F(\xi)=f\left(\|\xi\|^{2}\right), \quad \xi \in H,
$$

with some function $f$ on $[0, \infty)$. Since $F$ is infinitely many times Fréchet differentiable, $f$ is of $C^{\infty}$-class on $(0, \infty)$ and is continuous at 0 . With the help of Lemma 3.5 we have

$$
\Delta F(\xi)=2 f^{\prime}\left(\|\xi\|^{2}\right), \quad \xi \in H, \quad \xi \neq 0 .
$$

Suppose in addition that $F$ is an eigenfunction of $\Delta$ with eigenvalue $\lambda \in$ $\boldsymbol{R}$, i.e., $\Delta F=\lambda F$. Then by (3-6) we come to the equation:

$$
\lambda f(t)=2 f^{\prime}(t), \quad t>0 .
$$

The solution is given by $f(t)=C e^{\lambda t / 2}, t \geqslant 0$, for some $C \in \boldsymbol{R}$. Hence from (3-5) we obtain (3-4).

Q.E.D. 


\section{§4. A characterization of the Lévy Laplacian}

Having discussed in the previous section the structure of the rotation group $O(H ; \Delta)$ describing the invariance of the Lévy Laplacian, we now start on the problem of characterizing the Lévy Laplacian by means of the invariance. To our goal we recall the following properties:

(L1) $\Delta$ is a continuous linear operator from $\mathfrak{N}$ into itself (Proposition 1.4);

(L2) $\Delta$ is a derivation on $\mathfrak{R}$ (Proposition 1.5);

(L3) $\Delta$ annihilates linear functions, i.e., $\Delta F=0$ for any linear function $F(\xi)=\langle a, \xi\rangle, a \in H$;

(L4) for any non-negative quadratic function $F(\xi)=\langle A \xi$, $\xi\rangle$ with $A \in \mathscr{A}, \Delta F(\xi)$ is a non-negative constant;

(L5) $\Delta$ is invariant under $\mathfrak{I}(T, \nu)$ (Theorem A).

By definition (L3) and (L4) are apparent. Needless to say, properties (L2) and (L5) are particularly interesting. We note here that under (L1) and (L2) the statement (L3) is equivalent to the following

(L3') $\Delta$ annihilates cylindrical functions.

Here by a cylindrical function we mean a function $F$ of the form:

$$
F(\xi)=f\left(\left\langle a_{1}, \xi\right\rangle, \cdots,\left\langle a_{m}, \xi\right\rangle\right), \quad \xi \in H,
$$

where $f$ is a $C^{\infty}$-function on $\boldsymbol{R}^{m}$ and $a_{1}, \cdots, a_{m} \in H$.

The rest of this section will be devoted to the proof of the following

THEOREM B (Characterization of the Lévy Laplacian). If an operator on $\mathfrak{N}$ satisfies the properties (L1)-(L5), it is a constant multiple of the Lévy Laplacian.

Suppose that we are given an operator $\Lambda$ on $\mathfrak{N}$ satisfying the five properties (L1)-(L5).

Lemma 4.1. If $F$ is a quadratic function given by $F(\xi)=\langle K \xi, \xi\rangle$, $K \in \mathscr{K}$, then $\Lambda F(\xi)=0$ for any $\xi \in H$.

Proof. We may assume without loss of generality that $K$ is symmetric. Choose a sequence $\left\{K_{n}\right\}_{n=1}^{\infty}$ of finite rank operators such that $\left\|K_{n}-K\right\| \rightarrow 0$ and put $F_{n}(\xi)=\left\langle K_{n} \xi, \xi\right\rangle$. Then $F_{n}$ becomes a normal polynomial and $F_{n} \rightarrow F$ in $\mathfrak{R}$. Hence from (L1) we see that

$$
\lim _{n \rightarrow \infty} \Lambda F_{n}(\xi)=\Lambda F(\xi) .
$$


On the other hand, note that $F_{n}$ is a cylindrical function for $K_{n}$ is an operator of finite rank. We therefore see from $\left(\mathrm{L}^{\prime}\right)$ that $\Lambda F_{n}(\xi)=0$ for any $\xi \in H$. Consequently, $\Lambda F(\xi)=0$ by $(4-1)$.

Q.E.D.

We next consider a quadratic function

$$
F_{\varphi}(\xi)=\frac{1}{2}\langle M(\varphi) \xi, \xi\rangle, \quad \varphi \in L^{\infty}(T, \nu) .
$$

Since $\varphi$ can be written as difference of two non-negative functions in $L^{\infty}(T, \nu)$, it follows from (L4) that $\Lambda F_{\varphi}(\xi)$ is a constant which will be denoted by $\lambda(\varphi)$. Obviously, $\lambda$ becomes a continuous and non-negative linear functional on $L^{\infty}(T, \nu)$. While, for any $\alpha \in \mathfrak{T}(T, \nu)$ we have

$$
F_{\varphi \circ \alpha-1}=U\left(g_{\alpha}\right) F_{\varphi}
$$

Hence by (L5) we have

$$
\lambda\left(\varphi \circ \alpha^{-1}\right)=\Lambda F_{\varphi \circ \alpha-1}=\Lambda U\left(g_{\alpha}\right) F_{\varphi}=U\left(g_{\alpha}\right) \Lambda F_{\varphi}=\lambda(\varphi) .
$$

Namely, $\lambda$ is invariant under $\mathfrak{I}(T, \nu)$. We shall prove that such a functional is unique.

Propositron 4.2. Let $\lambda$ be a continuous and non-negative linear functional on $L^{\infty}(T, \nu)$. If $\lambda$ is invariant under $\mathfrak{I}(T, \nu)$, i.e., $\lambda\left(\varphi \circ \alpha^{-1}\right)=\lambda(\varphi)$ for any $\varphi \in L^{\infty}(T, \nu)$ and $\alpha \in \mathfrak{I}(T, \nu)$, then

$$
\lambda(\varphi)=c \int_{T} \varphi(t) d \nu(t), \quad \varphi \in L^{\infty}(T, \nu),
$$

with some constant $c \geqslant 0$.

For the proof we need the following

Lemma 4.3. Set $T=[0,1]$ and let $\nu$ the Lebesgue measure on $T$. Let $\lambda$ be a finitely additive set function on the Lebesgue measurable subsets such that (i) $\lambda$ is non-negative; (ii) $\lambda(S)=0$ for any $S$ with $\nu(S)=0$; (iii) $\lambda$ is invariant under $\mathfrak{I}(T, \nu)$. Then for any pair $a, b$ with $0 \leqslant a \leqslant b$ $\leqslant 1$ we have

$$
\lambda([a, b])=c(b-a)
$$

with $c=\lambda(T)$.

Proof. For any positive integers $n$ and $j$ with $1 \leqslant j \leqslant n$ there exists 
a measure-preserving transformation $\alpha_{j} \in \mathfrak{T}(T, \nu)$ such that $\alpha_{j}\left(\left[\frac{j-1}{n}, \frac{j}{n}\right]\right)$ $=\left[0, \frac{1}{n}\right]$. Hence $\lambda\left(\left[\frac{j-1}{n}, \frac{j}{n}\right]\right)=\frac{c}{n}$ with $c=\lambda(T)$. Therefore (4-3) is true for any pair of rational numbers $a, b$ with $0 \leqslant a<b \leqslant 1$. For the assertion we only need to approximate $a$ and $b$ by rational numbers and to note the non-negativity of $\lambda$.

Q.E.D.

Proof of Proposition 4.2. The functional $\lambda$ is canonically identified with a finitely additive set function on the $\sigma$-field $\mathscr{T}$ which is absolutely continuous with respect to $\nu$ (e.g., [6, Chap. IV, Theorem 8.16]). It is therefore sufficient to prove that

$$
\lambda(S)=c \nu(S)
$$

for any $S \in \mathscr{T}$ with $c=\lambda(T)$. First suppose that $S$ is an open subset of $T$. Then there is an isomorphism $\varphi$ from $T$ onto $[0,1]$ such that

(i) the image measure $\nu \circ \varphi^{-1}$ is the Lebesgue measure on $[0,1]$;

(ii) $\varphi(S)=[0, \nu(S))$.

It then follows from Lemma 4.2 that $\lambda(S)=c \nu(S)$. Hence (4-4) is valid for any open subset $S$ of $T$, and therefore, so is for any closed subset. Moreover, (4-4) is valid for any Borel subset $S$ of $T$. For there are sequences $\left\{F_{n}\right\}$ of closed subsets and $\left\{G_{n}\right\}$ of open subsets of $T$ such that $F_{1} \subset F_{2} \subset \cdots \subset S \subset \cdots \subset G_{2} \subset G_{1}$ with $\lim _{n \rightarrow \infty} \nu\left(F_{n}\right)=\lim _{n \rightarrow \infty} \nu\left(G_{n}\right)=\nu(S)$. Finally, (4-4) is true for any $S \in \mathscr{T}$ because every measurable subset $S \in \mathscr{T}$ coincides to a Borel subset modulo null sets and because $\lambda$ is absolutely continuous with respect to $\nu$.

Q.E.D.

LEMMA 4.4. Assume that an operator 1 satisfies the conditions (L1)(L5). Then there exists a constant $c \geqslant 0$ such that $\Lambda F=c \Delta F$ for any quadratic function $F$ of the form $F(\xi)=\langle A \xi, \xi\rangle$ with $A \in \mathscr{A}$.

Proof. Put $A=M(\varphi)+K$. Then by Lemma 4.1 and the discussion before Proposition 4.2 we see that $\Lambda F(\xi)=2 \lambda(\varphi)$. It follows immediately from Proposition 4.2 that

$$
\Lambda F(\xi)=2 c \int_{T} \varphi(t) d \nu(t)=c \Delta F(\xi) .
$$

This proves the assertion.

Q.E.D.

Proof of Theorem B. Since $\Lambda$ is a derivation by assumption (L2), it 
annihilates constants as well as the Lévy Lapalcian 4 . Taking (L3) and Lemma 4.4 into account, we see that

$$
\Lambda F=c \Delta F
$$

for any normal polynomial $F$ of degree $\leqslant 2$. Using (L2) again, we see easily that (4-5) is true for arbitrary normal polynomials. Finally the property (L1) implies that $(4-5)$ is also valid for any normal function $F \in \mathfrak{N}$ because the normal polynomials form a dense subspace of $\mathfrak{N}$.

Q.E.D.

\section{§5. The Lévy Laplacian in terms of spherical mean}

In this section we discuss another approach to the Lévy Laplacian in terms of spherical mean due to Lévy. This approach is also interesting to discuss certain problems of stochastic calculus. In addition, we shall observe that the notion of normal functions is quite natural from this viewpoint as well.

For each $n=1,2, \cdots$, the unit sphere $S^{n-1} \subset \boldsymbol{R}^{n}$ is regarded as a compact subset of $H$ by means of the mapping:

$$
h=\left(h_{1}, \cdots, h_{n}\right) \longmapsto \sum_{k=1}^{n} h_{k} e_{k} \in H, \quad h \in S^{n-1} .
$$

We denote by $d S_{n-1}(h)$ the normalized uniform measure on $S^{n-1}$. Then the (asymptotic) spherical mean of a function $F$ over the sphere of radius $\rho \in \boldsymbol{R}$ with center at $\xi \in H$ is defined by

$$
M F(\xi, \rho)=\lim _{n \rightarrow \infty} \int_{S^{n-1}} F(\xi+\rho h) d S_{n-1}(h)
$$

provided the limit exists. We shall prove the following

Proposition 5.1. Every normal function $F \in \mathfrak{N}$ admits the spherical mean $\operatorname{MF}(\xi, \rho)$ for any $\xi \in H$ and $\rho \in R$, and the identity

$$
\Delta F(\xi)=2 \lim _{\rho \rightarrow 0} \frac{M F(\xi, \rho)-F(\xi)}{\rho^{2}}
$$

holds.

LEMMA 5.2. Let $F_{j}$ be a quadratic function given by $F_{j}(\xi)=\left\langle A_{j} \xi, \xi\right\rangle$ with $A_{j} \in \mathscr{A}, j=1,2, \cdots, q$, and put $F(\xi)=\prod_{j=1}^{q} F_{j}(\xi)$. Then

$$
M F(0, \rho)=\prod_{j=1}^{q} M F_{j}(0, \rho), \quad \rho \in \boldsymbol{R} .
$$


Proof. We define a symmetric $p$-form $\hat{F}$ on $H$ with $p=2 q$ by

$$
\hat{F}\left(\xi_{1}, \cdots, \xi_{p}\right)=\frac{1}{p !} \sum_{\sigma \in \mathbb{E}_{p}} \prod_{j=1}^{q}\left\langle A_{j} \xi_{\sigma(2 j-1)}, \xi_{\sigma(2 j)}\right\rangle
$$

where $\widetilde{S}_{p}$ stands for the permutation group of $p$ symbols. Then $F(\xi)=$ $\hat{F}(\xi, \cdots, \xi)$ and

$$
M F(0, \rho)=\rho^{p}(p-1) ! ! \lim _{n \rightarrow \infty} n^{-q} \sum_{i_{1}, \ldots, i_{q}=1}^{n} \hat{F}\left(e_{i_{1}}, e_{i_{1}}, \cdots, e_{i_{q}}, e_{i_{q}}\right)
$$

This formula is due to [26, Lemma 3.1]. In view of (5-3) we have

$$
\begin{aligned}
n^{-q} & \sum_{i_{1}, \cdots, i_{q}=1}^{n} \hat{F}\left(e_{i_{1}}, e_{i_{1}}, \cdots, e_{i_{q}}, e_{i_{q}}\right) \\
& =\frac{q ! 2^{q}}{p !} \cdot n^{-q} \sum_{i_{1}, \cdots, i_{q}=1}^{n}\left\langle A_{1} e_{i_{1}}, e_{i_{1}}\right\rangle \cdots\left\langle A_{q} e_{i_{q}}, e_{i_{q}}\right\rangle+R .
\end{aligned}
$$

Here $R$ is a sum of finitely many $\left(p !-q ! 2^{q}\right)$ terms of the form:

$$
\frac{n^{-q}}{p !} \sum_{i_{1}, \cdots, i_{q}=1}^{n}\left\langle A_{1} e_{i_{1}}, e_{j_{1}}\right\rangle \cdots\left\langle A_{q} e_{i_{q}}, e_{j_{q}}\right\rangle
$$

where $\left(j_{1}, \cdots, j_{q}\right)=\left(i_{\sigma(1)}, \cdots, i_{\sigma(q)}\right)$ for some $\sigma \in \mathbb{S}_{q}, \sigma \neq e$. Using the Schwarz inequality we have

$$
\frac{n^{-q}}{p !}\left|\sum_{i_{1}, \ldots, i_{q}=1}^{n}\left\langle A_{1} e_{i_{1}}, e_{j_{1}}\right\rangle \cdots\left\langle A_{q} e_{i_{q}}, e_{j_{q}}\right\rangle\right| \leqslant \frac{n^{-1 / 2}}{p !}\left\|A_{1}\right\| \cdots\left\|A_{q}\right\|
$$

and therefore,

$$
|R| \leqslant\left(p !-q ! 2^{q}\right) \frac{n^{-1 / 2}}{p !}\left\|A_{1}\right\| \cdots\left\|A_{q}\right\| \longrightarrow 0 \quad \text { as } n \longrightarrow \infty .
$$

Consequently,

$$
\begin{aligned}
M F(0, \rho) & =\rho^{p}(p-1) ! ! \frac{q ! 2^{q}}{p !} \lim _{n \rightarrow \infty} n^{-q} \sum_{i_{1}, \ldots, i_{q}=1}^{n}\left\langle A_{1} e_{i_{1}}, e_{i_{1}}\right\rangle \cdots\left\langle A_{q} e_{i_{q}}, e_{i_{q}}\right\rangle \\
& =\rho^{p} L\left(A_{1}\right) \cdots L\left(A_{q}\right),
\end{aligned}
$$

where $L$ is defined by (1-6). Since $M F_{j}(0, \rho)=\rho^{2} L\left(A_{j}\right)$, we obtain (5-2).

Q.E.D.

Proof of Proposition 5.1. Evidently, $M F(0, \rho)=0$ if $F$ is a homogeneous polynomial of odd degree. Using this fact we see that the spherical mean $M F(0, \rho)$ always exists for any normal polynomial and that (5-2) is 
also valid for any normal polynomials $F_{1}, \cdots, F_{q}$. Moreover, this is true for any normal function because every normal function can be approximated by normal polynomials uniformly on the sphere of radius $\rho$ with center at 0 . Note that, for any $F \in \mathfrak{N}$ the translated function $V(\eta) F$ is also normal for any $\eta \in H$ (cf. (3-3)). Moreover, it is obvious from the definition that $M(V(\eta) F)(\xi, \rho)=M F(\xi-\eta, \rho)$. Hence, we conclude that $M F(\xi, \rho)=M(V(-\xi) F)(0, \rho)$ exists. Since $\Delta F(\xi)$ exists by Lemma 1.3, we obtain the identity (5-1) with the help of the result [26, Proposition 1.1].

Q.E.D.

Corollary 5.3. For any pair of normal functions $F_{1}$ and $F_{2}$ we have

$$
M\left(F_{1} F_{2}\right)(\xi, \rho)=M F_{1}(\xi, \rho) \cdot M F_{2}(\xi, \rho), \quad \xi \in H, \quad \rho \in R .
$$

Proof. During the proof of Proposition 5.1 we have already established that the assertion is true for $\xi=0$. For an arbitrary $\xi \in H$ we need only to apply the translation operator $V(-\xi)$.

Q.E.D.

Remark 5.4. For a quadratic function $F(\xi)=\langle A \xi, \xi\rangle$ with $A \in \mathscr{A}$ it holds that $\operatorname{MF}(\xi, \rho)=F(\xi)+\rho^{2} L(A)$. From this we see that a quadratic function possesses the mean value property if and only if it is harmonic with respect to the Lévy Laplacian (see also [26]).

Remark 5.5. There is an interesting relation between the Lévy Laplacian and another infinite dimensional Laplacian introduced by Gross [8]. The latter will be called the Gross Laplacian and defined as

$$
\Delta_{G} F(\xi)=\text { Trace } F^{\prime \prime}(\xi), \quad \xi \in H
$$

if $F^{\prime \prime}(\xi)$ is an operator of trace class. Then we may prove with no difficulty that

$$
\Delta F(\xi)=\lim _{N \rightarrow \infty} \frac{1}{N} \Delta_{G}\left(F \circ I_{N}\right)(\xi), \quad F \in \mathfrak{R},
$$

where $I_{N}$ is an integral operator on $H$ with integral kernel

$$
K_{N}(s, t)=\sum_{n=1}^{N} e_{n}(s) e_{n}(t) .
$$

In this connection, see also [20]. 


\section{Appendix. Construction of equally dense complete orthonormal sequences}

As is easily expected, generally speaking, the property of a CONS being equally dense depends upon the arrangement. In the first half of this appendix we construct CONS's which are equally dense independent of the arrangement. For technical simplicity we specialize our considerations to the case where $T=[0,1]$ equipped with the Lebesgue measure.

Let $G$ be the countably infinite direct product of the identical additive group $Z_{2}=\{0,1\}$ :

$$
G=\left\{g=\left(\tilde{i}_{1}, \tilde{\imath}_{2}, \cdots\right) ; \tilde{r}_{n} \in \boldsymbol{Z}_{2}\right\} .
$$

Equipped with the product topology, $G$ becomes a compact abelian group. We denote by $\nu$ the normalized Haar measure of $G$. The binary expansion yields an isomorphism (in the sense of measure theory) from $G$ onto $[0,1]$ by means of the mapping:

$$
\beta: g=\left(\gamma_{1}, \gamma_{2}, \cdots\right) \longmapsto \sum_{n=1}^{\infty} \frac{\gamma_{n}}{2^{n}} \in[0,1], \quad g \in G .
$$

The image of the Haar measure $\nu$ under $\beta$ coincides with the Lebesgue measure.

It is known that the dual group $G^{\wedge}$ becomes a CONS in $L^{2}(G, \nu)$ (see e.g., [11, Remarks 27.4 and Theorem 27.40]). Since every $f \in G^{\wedge}$ and therefore $f \circ \beta^{-1}$ takes values in $\{ \pm 1\}$, we have

Proposition A.1. The set $\left\{f \circ \beta^{-1} ; f \in G^{\wedge}\right\}$ is an equally dense CONS independent of the arrangement.

An equally dense CONS is also obtained from differential equations.

Proposition A.2. Let $r=r(t)$ be a continuous function on $[0,1]$. Denote by $u_{n}=u_{n}(t)$ the normalized $n$-th eigenfunction of the following second order differential equation:

$$
u^{\prime \prime}(t)-r(t) u(t)+\lambda u(t)=0
$$

with the boundary condition: $u(0)=u(1)=0$. Then $\left\{u_{n}\right\}_{n=1}^{\infty}$ becomes an equally dense CONS in $H=L^{2}(0,1)$ independent of the arrangement.

Proof. It is sufficient to show that

$$
\lim _{N \rightarrow \infty} \frac{1}{N} \sum_{n=1}^{N} \int_{0}^{1} \varphi(t) u_{g(n)}(t)^{2} d t=\int_{0}^{1} \varphi(t) d t
$$


for any $\varphi \in L^{\infty}(0,1)$ and $g \in \operatorname{Aut}(N)$, where $\operatorname{Aut}(N)$ stands for the group of all permutations on the set $N=\{1,2, \cdots\}$. For simplicity we put

$$
e_{n}(t)=\sqrt{2} \sin \pi n t \text { and } u_{n}(t)=e_{n}(t)+v_{n}(t) .
$$

With the help of the Riemann-Lebesgue theorem we may prove that $\left\{e_{g(n)}\right\}_{n=1}^{\infty}$ is an equally dense CONS for any $g \in \operatorname{Aut}(N)$. We then need only to show that

$$
\lim _{N \rightarrow \infty} \frac{1}{N} \sum_{n=1}^{N} \int_{0}^{1} \varphi(t) u_{g(n)}(t)^{2} d t=\lim _{N \rightarrow \infty} \frac{1}{N} \sum_{n=1}^{N} \int_{0}^{1} \varphi(t) e_{g(n)}(t)^{2} d t .
$$

But this is immediate from the following inequalities:

$$
\begin{aligned}
& \left|\int_{0}^{1} \varphi(t) e_{n}(t) v_{n}(t) d t\right| \leqslant \frac{\sqrt{2} M\|\varphi\|_{\infty}}{n} \\
& \left|\int_{0}^{1} \varphi(t) v_{n}(t)^{2} d t\right| \leqslant \frac{M^{2}\|\varphi\|_{\infty}}{n^{2}},
\end{aligned}
$$

where $M>0$ is a constant such that $\left|v_{n}(t)\right| \leqslant M / n$ for all $t \in[0,1]$. For the existence of such a constant, see [2, Chapter V, § 11].

Q.E.D.

Finally we make two remarks on relation between equally dense CONS's and infinite dimensional rotation groups.

Proposition A.3. If $\left\{e_{n}\right\}_{n=1}^{\infty}$ is an equally dense CONS, so is $\left\{g e_{n}\right\}_{n=1}^{\infty}$ for any $g \in O(H ; \Delta)$.

Proof. According to Theorem A, any $g \in O(H ; \Delta)$ is factorized as $g=g_{\alpha} g_{1} g_{2}$ with $\alpha \in \mathfrak{T}(T, \nu), \quad g_{1} \in O_{a}(H)$ and $g_{2} \in O_{c}(H)$. Then, for any $\varphi \in L^{\infty}(T, \nu)$ we have

$$
\left\langle M(\varphi) g e_{n}, g e_{n}\right\rangle=\left\langle g^{-1} M(\varphi) g e_{n}, e_{n}\right\rangle=\left\langle M(\varphi \circ \alpha) e_{n}, e_{n}\right\rangle+\left\langle K e_{n}, e_{n}\right\rangle
$$

with some $K \in \mathscr{K}$. Hence

$$
\lim _{N \rightarrow \infty} \frac{1}{N} \sum_{n=1}^{N}\left\langle M(\varphi) g e_{n}, g e_{n}\right\rangle=\int_{T} \varphi(\alpha(t)) d \nu(t)=\int_{T} \varphi(t) d \nu(t) .
$$

This proves that $\left\{g e_{n}\right\}_{n=1}^{\infty}$ is also equally dense.

Q.E.D.

On the other hand, it is interesting to consider permutations which preserve the property of a CONS being equally dense. Following [23] we introduce the Lévy group:

$$
\mathscr{G}=\left\{g \in \operatorname{Aut}(N) ; \lim _{N \rightarrow \infty} \frac{1}{N}|\{1 \leqslant n \leqslant N ; g(n)>N\}|=0\right\} .
$$


Then the following result is easy to see.

Proposition A.4. If $\left\{e_{n}\right\}_{n=1}^{\infty}$ is an equally dense CONS, so is the rearranged CONS $\left\{e_{g(n)}\right\}_{n=1}^{\infty}$ for any $g \in \mathscr{G}$.

This suggests some similarity of equally dense CONS's and uniformly distributed sequences, in this connection see [25].

\section{REFERENCES}

[1] Balakrishnan, A. V., A white noise version of the Girsanov formula, in: Itô, K. (ed.) Proceedings of the international symposium on stochastic differential equations, Kyoto, 1976, pp. 1-19. Kinokuniya, Tokyo, 1978.

[2] Courant, R., Hilbert, D., Methods of mathematical physics, Vol. 1 (1st Eng. ed.), Interscience, New York, 1953.

[ 3 ] Dixmier, J., Von Neumann algebras, North-Holland, Amsterdam-New York-Oxford, 1981.

[ 4 ] — $C^{*}$-Algebras, North-Holland, Amsterdam-New York-Oxford, 1982.

[5] Dorfman, I. Ya., On means and the Laplacian of functions on Hilbert space, Math. USSR Sbornik, 10 (1970), 181-196.

[6] Dunford, N., Schwartz, J. T., Linear operators, Part I: General theory, Wiley, New York, 1988.

[7] Feller, M. N., Infinite dimensional elliptic equations and operators of Lévy type, Russian Math. Surveys, 41-4 (1986), 119-170.

[ 8 ] Gross, L., Potential theory on Hilbert space, J. Funct. Anal., 1 (1967), 123-181.

[9] Halmos, P. R., von Neumann, J., Operator methods in classical mechanics, II, Ann. of Math., 43 (1942), 332-350.

[10] Hasegawa, Y., Lévy's functional analysis in terms of an infinite dimensional Brownian motion I-III, Osaka J. Math., 19 (1982), 405-428; 549-570; Nagoya Math. J., 90 (1983), 155-173.

[11] Hewitt, E., Ross, K. A., Abstract harmonic analysis II, Springer-Verlag, BerlinHeidelberg-New York, 1970.

[12] Hida, T., Analysis of Brownian functionals, Carleton Math. Lect. Notes Vol. 13, 1975. 2nd ed., 1978.

[13] _ Brownian motion, Applications of Mathematics Vol. 11, Springer-Verlag, New York-Heidelberg-Berlin, 1980.

[14] - Brownian motion and its functionals, Ricerche Mat., 34 (1985), 183-222.

[15] —- Analysis of Brownian functionals, Lecture Notes, IMA, University of Minnesota, 1986.

[16] - Saitô, K., White noise analysis and the Lévy Laplacian, in: Albeverio, S., Blanchard, Ph., Hazewinkel, M., Streit, L. (eds.) Stochastic processes in physics and engineering. pp. 177-184. D. Reidel Pub. Co., Dordrecht-Boston-LancasterTokyo, 1988.

[17] Johnson, B. E., Parrott, S. K., Operators commuting with a von Neumann algebra modulo the set of compact operators, J. Funct. Anal., 11 (1972), 39-61.

[18] Kubo, I., Takenaka, S., Calculus on Gaussian white noise II, Proc. Japan Acad., 56A (1980), 411-416.

[19] Kuo, H.-H., On Laplacian operators of generalized Brownian functionals, in: Itô, K., Hida, T. (eds.) Stochastic processes and their applications. Proceedings, Nagoya, 1985 (Lect. Notes Math., vol. 1203), pp. 119-128. Springer-Verlag, Berlin- 
Heidelberg-New York, 1986.

[20] — Obata, N., Saitô, K., Lévy Laplacian of generalized functions on a nuclear space, to appear in J. Funct. Anal. (1990).

[21] Lévy, P., Leçons d'analyse fonctionnelle, Gauthier-Villars, Paris, 1922.

[22] — - Problèmes concrets d'analyse fonctionnelle, Gauthier-Villars, Paris, 1951.

[23] Obata, N., A note on certain permutation groups in the infinite dimensional rotation group, Nagoya Math. J., 109 (1988), 91-107.

[24] —- Analysis of the Lévy Laplacian, Soochow J. Math., 14 (1988), 105-109.

[25] - Density of natural numbers and the Lévy group, J. Number Theory, 30 (1988), 288-297.

[26] — The Lévy Laplacian and mean value theorem, in: Heyer, H. (ed.) Probability measures on groups IX. Proceedings, Oberwolfach, 1988 (Lect. Notes Math., vol. 1379), pp. 242-253. Springer-Verlag, Berlin-Heidelberg-New York, 1989.

[27] Shilov, G. E., On some questions of analysis in Hilbert space, I-III. Funct. Anal. Appl., 1 (1967), 158-165; Amer. Math. Soc. Transl. (2), 90 (1970), 1-16; Math. USSR Sbornik, 3 (1967), 153-158.

[28] von Neumann, J., Einige Sätze über messbare Abbildungen, Ann. of Math., 33 (1932), 574-586.

[29] Yoshizawa, H., Rotation group of Hilbert space and its application to Brownian motion, in: Proceedings of the international conference on functional analysis and related topics, Tokyo, 1969, pp. 414-423. Univ. of Tokyo Press, 1970.

Department of Mathematics

School of Science

Nagoya University

Nagoya, 464-01, Japan 